\title{
Molecular recognition of rhodopsin kinase GRK1 and recoverin is tuned by switching intra/intermolecular electrostatic interactions
}

Seher Abbas, ${ }^{1}$ Valerio Marino, ${ }^{2,3}$ Daniele Dell'Orco, ${ }^{2}$ Karl-Wilhelm Koch,,${ }^{{ }^{*}}$

${ }^{1}$ Department of Neuroscience, Division of Biochemistry, University of Oldenburg, 26111 Oldenburg, Germany

${ }^{2}$ Department of Neurosciences, Biomedicine and Movement Sciences, Section of Biological Chemistry, University of Verona, 37134 Verona, Italy

${ }^{3}$ Department of Translational Research and New Technologies in Medicine and Surgery, University of Pisa, 56126 Pisa, Italy

\section{Supplementary Information}

Table S1

\begin{tabular}{|c|c|c|c|c|}
\hline Name & ZD-s & $\begin{array}{c}\text { Native-like } \\
\text { solutions }\end{array}$ & $\Delta$ ZD-s & $\Delta \mathbf{\Delta G}^{\mathbf{0}}(\mathbf{k c a l} / \mathbf{m o l})$ \\
\hline WT & $36.0 \pm 0.20$ & 444 & - & - \\
\hline K192E & $35.6 \pm 0.19$ & 441 & -0.4 & 0.73 \\
\hline K192L & $35.5 \pm 0.18$ & 437 & -0.5 & 0.82 \\
\hline
\end{tabular}

ZD-s is the ZDOCK 2.3 score presented as average \pm std. err, $\Delta \Delta \mathrm{G}^{0}$ is calculated with respect to the WT as elucidated in the Methods. 

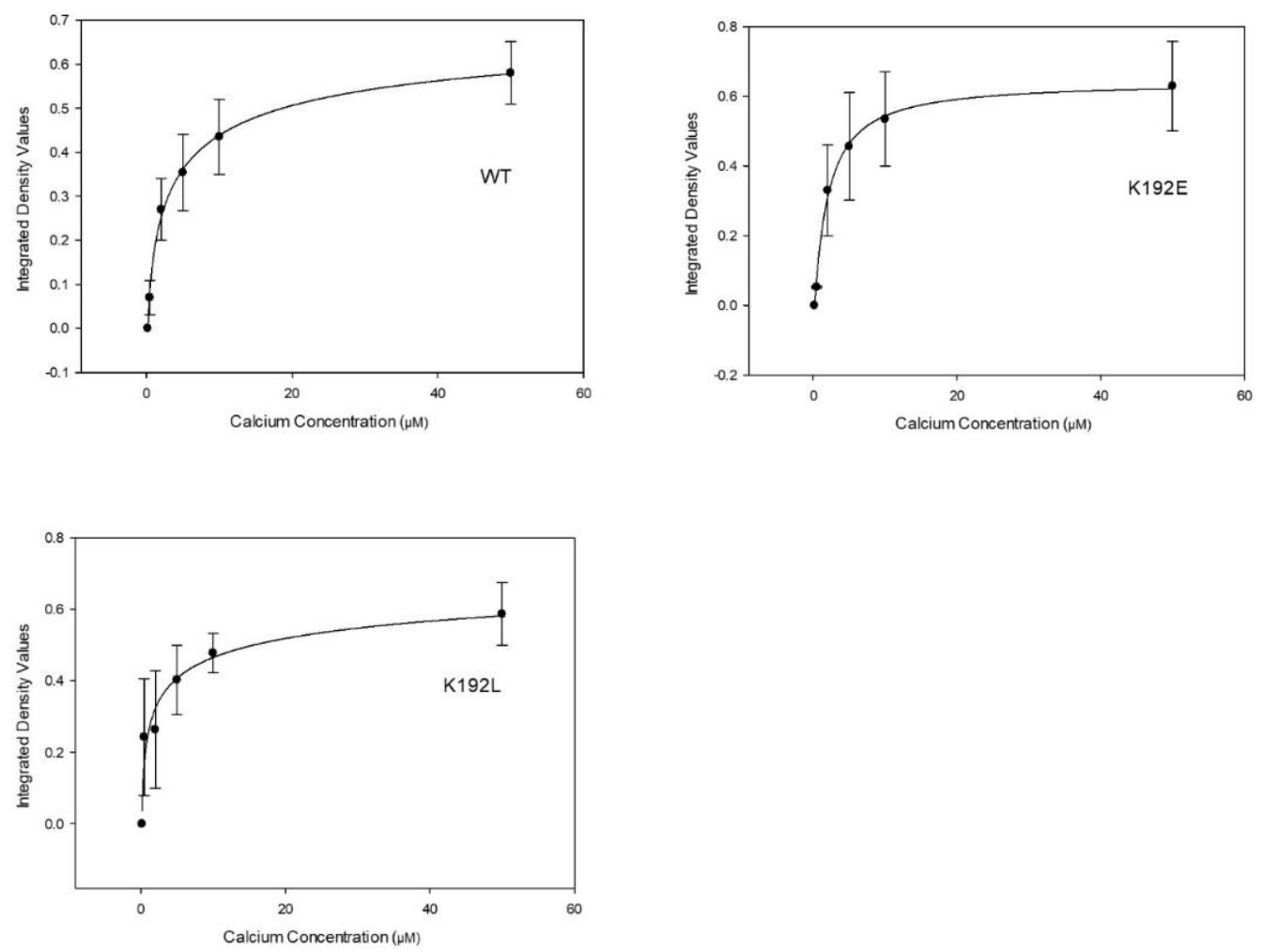

Figure S1. Recoverin binding to membranes. All myristoylated and non-myristoylated Rec variants were tested for membrane binding in the presence and absence of $\mathrm{Ca}^{2+}\left(5 \mathrm{mM}\right.$ EGTA for $\mathrm{Ca}^{2+}$-free conditions). Both mutants K192L and K192E showed a binding pattern similar to that of the WT (Table 1).

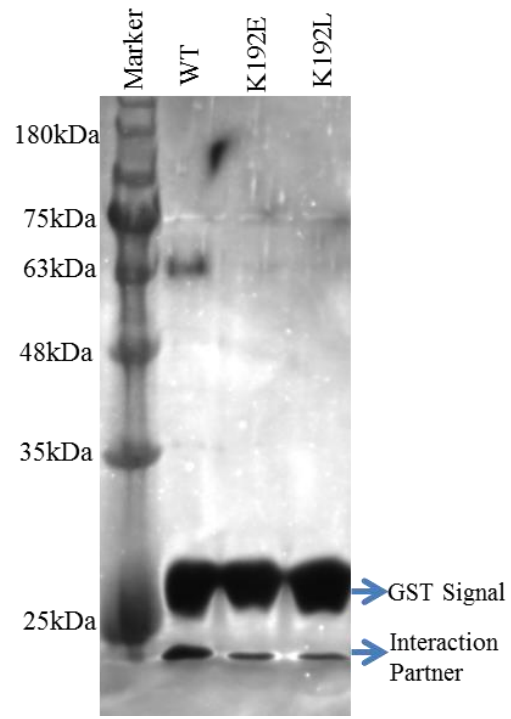

Figure S2. Interaction of Rec variants K192E and K192L with GRK1 monitored by pull down assay. Both Rec mutants showed weaker binding to the N-terminus of the kinase than the WT. All Rec variants were tested in the presence of $2 \mathrm{mM} \mathrm{CaCl}_{2}$. Eluted proteins were tested in two independent western blots using anti Rec and anti GST antibodies. 

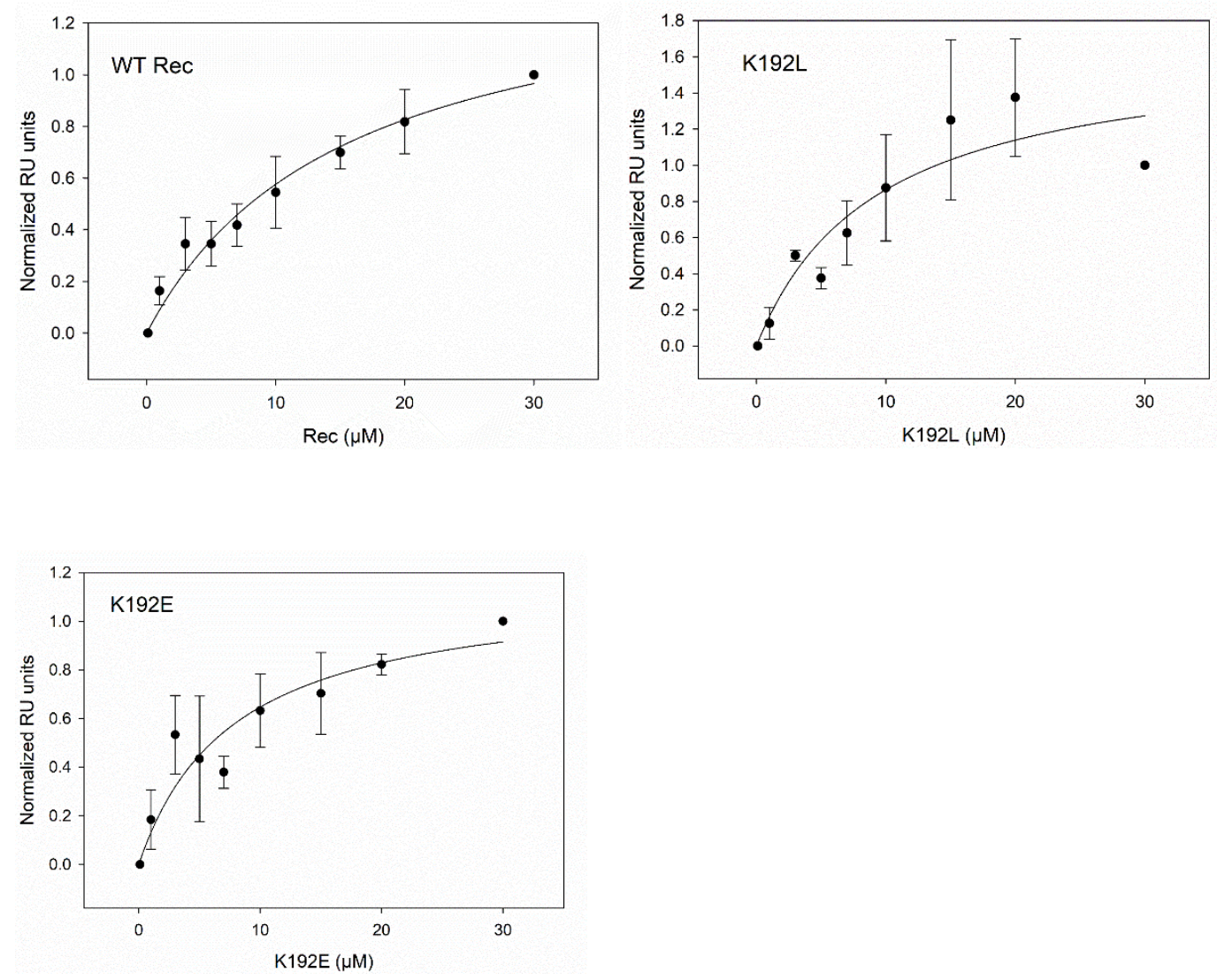

Figure S3. Interaction of Rec variants (WT, mutants K192L and K192E) with immobilized GSTGRK1 monitored by surface plasmon resonance spectroscopy. Normalized RU values were calculated as described in the Methods section. Titrations (two in each panel) are representative of a total of $n=4$ that were used for the calculation of parameters in Table 1 (main text). 